\title{
Put that Hologram there - Probing Mobile Interaction Experiences for a Vision of Mixed Material Public Spaces
}

\author{
Ilhan Aslan, Chi Tai Dang, Ruben Schlagowski, Michael Dietz, Fabian Brain and Elisabeth André \\ lastname@hcm-lab.de \\ Human-Centered Multimedia Lab, Augsburg University \\ Augsburg, Germany
}

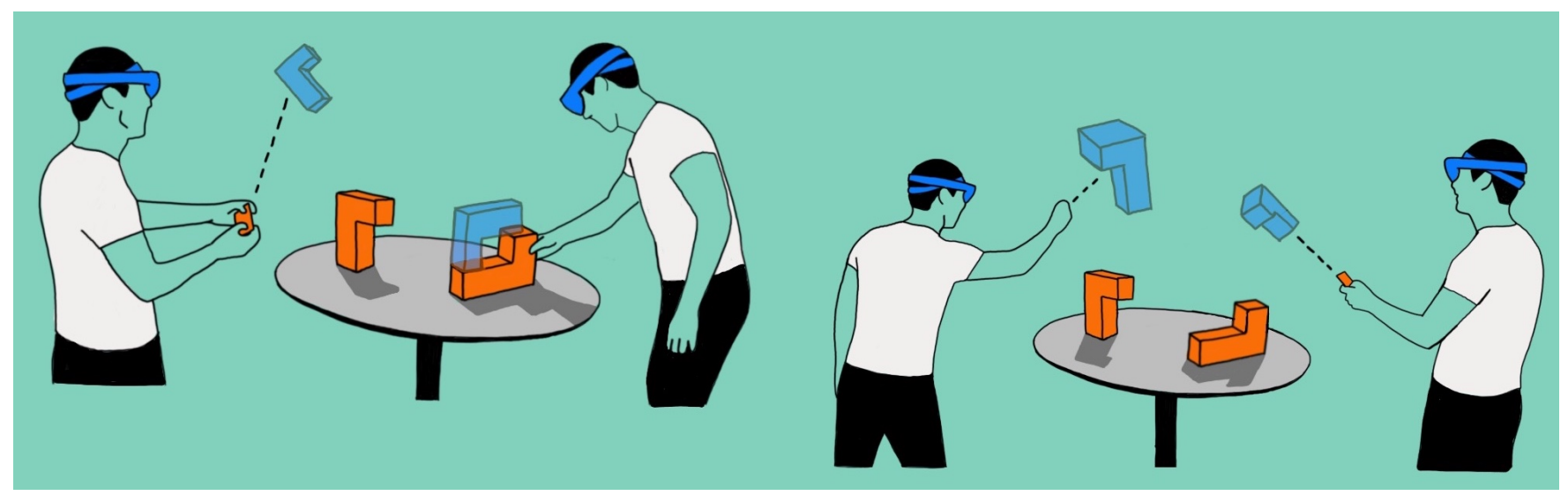

Figure 1: Overview of interaction techniques for manipulating a "hologram" (with mid-air gestures or with touch and/or tilt input with a mobile) and a real object in order to position them next to each other in a predefined way.

\begin{abstract}
The materiality of urban spaces has been changing by progress and consequently the way we, as townsmen or visitors, interact in these spaces is changing. For example, within the last decade using a mobile digital bus ticket has become a common practice in many cities around the world. Contemporary progress in mobile augmented reality technologies has already introduced novel pervasive displays, such as Microsoft's HoloLens, which allows to display mixed realities containing tangible real and "intangible" holographic objects. Now, we seem to be at a crossroads, living in an increasingly digital urban space, which may soon turn into a mixed material space and transform in the process radically the materiality of urban spaces and interactions with objects in these spaces. In order to allow prototyping and probing such seemingly near-future (urban) interaction experiences, we built a system, which combines a mobile phone with Microsoft's HoloLens and enables exemplary interaction techniques to manipulate "holograms" by combining the capabilities of both these personal and mobile devices. In this paper, we first describe details of this system and then report on a
\end{abstract}

Permission to make digital or hard copies of all or part of this work for personal or classroom use is granted without fee provided that copies are not made or distributed for profit or commercial advantage and that copies bear this notice and the full citation on the first page. Copyrights for components of this work owned by others than the author(s) must be honored. Abstracting with credit is permitted. To copy otherwise, or republish, to post on servers or to redistribute to lists, requires prior specific permission and/or a fee. Request permissions from permissions@acm.org.

IoT '19, October 22-25, 2019, Bibao, Spain

(C) 2019 Copyright held by the owner/author(s). Publication rights licensed to ACM. ACM ISBN todo... \$todo

https://doi.org/todo study with 12 participants probing participants' experiences and expectations of a future urban mixed material space. Results of a thematic analysis highlight a two-sided view, in which despite some "fears" of radical change, which may cause a disparity of what (materials) matter more, participants demonstrated a desire to benefit from material complementarity.

\section{CCS CONCEPTS}

- Human-centered computing $\rightarrow$ Mixed / augmented reality.

\section{KEYWORDS}

HoloLens, mobile HCI, mixed reality, materiality

ACM Reference Format:

Ilhan Aslan, Chi Tai Dang, Ruben Schlagowski, Michael Dietz, Fabian Brain and Elisabeth André. 2019. Put that Hologram there - Probing Mobile Interaction Experiences for a Vision of Mixed Material Public Spaces. In International Conference on the Internet of Things (IoT '19), October 22-25, 2019, Bilbao, Spain. ACM, New York, NY, USA, 6 pages. https://doi.org/todo

\section{INTRODUCTION}

The change of urban spaces over time may be most obvious in (visually) present material objects and physical spaces. For example, it may be due to the industrial revolution that some cities integrated railways into their infrastructure and additional signs and objects were added continuously to the scenery to guide interactions of townsmen and visitors. Today, we seem to be midst another revolution, a digital revolution, which might change the face of urban space in new and unprecedented ways. Some change may have 
been process oriented and mostly invisible, such as more and more people traveling with digital public transportation tickets, which are carried and interacted with on mobiles. But progress in mobile and pervasive displays are signs for a next wave of changes, which will allow digital things with their functionalities to be embodied side by side with real things. Already, the late Professor Marshall McLuhan [18](26:09) has referred to the "hologram" as a mass media, which might follow television and be potentially "worse" than television in terms of its disruptive impact on society, since holograms can go completely around users.

Indeed, we as researchers and potential technology enthusiasts, may have observed with joy and concern the hype associated with "Pokemon Go" and players running around with their mobiles in theirs hands in search for invisible monsters. Today's media scientists who are exploring future mixed reality scenarios may also be aware of the artistic and critical design work of Keiichi Matsuda, who has been successful in drawing dark future visions, including "hyperpervasive" displays and mixed realities, and how they may cause overwhelming future personal experiences in urban spaces.

What makes "holograms" (immersive visual digital mixed reality objects) special as new "media" is how seamless they seem to fit in real spaces with their capabilities of spatio-visually representing (3D) content, including real objects. Janlert and Stolterman [20] mention holograms as examples for interfaces with conditional presence, and thus, as one of many new techniques to address the interface bottleneck, which has become a serious issue with the increase of digitalization. Some readers my correctly criticize our use of the term hologram, since real holograms would not require any viewing device. But please indulge our use of the term, while technically imprecise, the use of the term improves readability and helps in conveying future interaction experiences that most readers will immediately be able to associated with holograms.

In this paper, our aim is to study a specific near-future vision in which people will have interactive encounters with holograms and people will have personal mobile technology at their disposal for personal interaction with holograms. In order to probe, how users would experience interacting in such a scenario we built a system and a setup, and conducted a study with 12 participants. We asked participants to put a hologram next to a physical counterpart and put them into a closed shape (see Figure 1). We implemented three different modalities to interact in such a setting, namely (i) by using mid-air pinch gestures (ii) by touching/gesturing on the touch screen of the mobile display, and (iii) by touching the touch screen of the mobile and simultaneously tilting the mobile.

The research contribution of this paper is three-fold. First we present in detail how we implemented the system, which combines the HoloLens with mobile devices by utilizing a typical internet of things (IoT) messaging protocol and supports different modalities to manipulate holograms. Second, we present results of the user study regarding the usage of the different interaction modalities. Last but not least, results of a detailed qualitative thematic analysis is presented, including the proposition of the two reoccurring and interrelated main themes (i) Experiencing material complementarity (ii) and a disparity of what is virtual and what is real, which seem to exist across anticipated application contexts and my potentially introduce contradicting experiences.

\section{BACKGROUND}

The augmented reality technologies that we employed realizes mixed realities, which, according to Milgram's [14] continuum, lies in the space between purely real environments and purely virtual environments. Such technologies show the real environment to the user, however, augmented or superimposed with virtual objects creating the impression that the virtual objects coexist with real objects in the real world. In this work, we employ the optical-seethrough device $[11,21]$ HoloLens $^{1}$, which is a head-mounted display manufactured by Microsoft.

Head-mounted displays (HMD) were the first class of augmented reality devices proposed in literature and were initially demonstrated by Ivan Sutherland [21] in 1968. The HMD HoloLens is realized as a binocular optical-see-through device where semitransparent holographic lenses are placed in front of the user's eyes. Due to the partially transparent nature of these lenses, users can directly perceive the real world through them [3]. Since they are also partially reflective, the virtual objects can be projected onto the lenses so that the user sees a combination of the real world and the virtual image. In theory, this leads to a more natural perception experience compared to the presentation of the scene on a display [4].

There is a large amount of research for augmented reality with smartphones mostly based on the video-see-through approach. Our work, however, employs a head-mounted display to augment the environment and utilize a smartphone as tool to interact with the augmented reality. Waldow et al. [22] and Lee et al. [12] explored this combination and demonstrated that touch-based input on a smartphone tend to perform significantly better than in-air gestures for object manipulation in the augmented reality. However, in-air gestures were perceived more natural and were more likable than touch-input on smartphones.

Millette [15] also employed the combination of HoloLens and smartphone for a CAD application. Users may switch between a traditional desktop display with precise mouse input and the HoloLens with smartphone in-air tracking for 3D modeling tasks. They investigated novel bi-manual interaction techniques within the augmented reality and showed that both input modalities can be optimally used for different tasks within 3D modeling application.

Yu et al. [23] presented a system combining the HoloLens and a smartphone for placement of virtual objects with strong focus on geometry awareness and the technical aspects of mapping between real world and virtual world.

The work at hand complements existing related work by building on the (i) combination of HoloLens and smartphone based interaction techniques, but (ii) focusing on a qualitative analysis with a materiality stance towards an interaction space in which tangible and intangible objects coexist. A turn to materiality (e.g., [6, 9] has become a contemporary development in $\mathrm{HCI}$, and one could argue that it follows up previous turns, as described by Yvonne Rogers [19], such as the turn to embodiment [5]. We do this, believing that a materiality inspired stance will provide additional insights and help thoughtfully shape future urban spaces.

$\overline{{ }^{1} \text { https://www.microsoft.com/hololens }}$ 


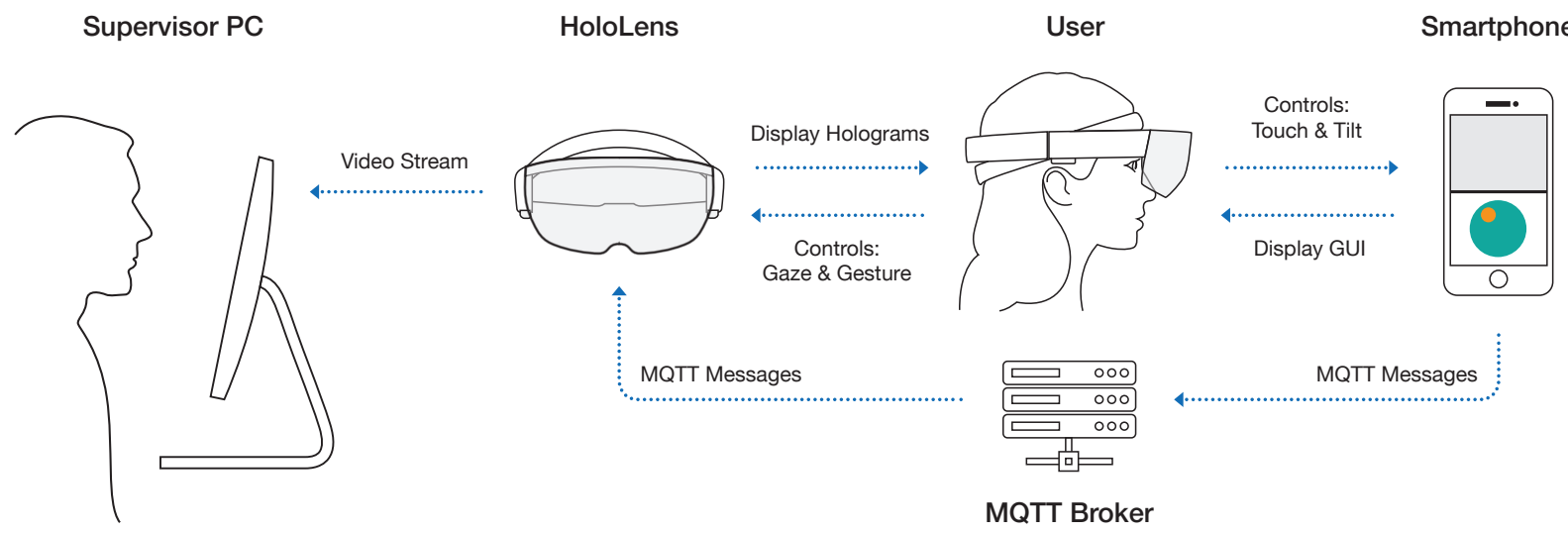

Figure 2: Technical setup of the prototype used in the user study

\section{PROTOTYPE}

The prototype combines the Augmented Reality HMD HoloLens running a Unity3d app with a custom-built mobile app for Android OS on a smartphone. Figure 2 illustrates all components included in the prototype and show how they communicate/interact with each other. For the user study, an additional PC was used to be able to observe participants' interactions with holograms via a live stream, displaying in real-time the augmented view that participant's perceive while wearing the AR HMD. For communication between the mobile app and HoloLens, the Message Queuing Telemetry Transport (MQTT) protocol was used. MQTT is a popular protocol for interconnecting networked IoT devices.

\subsection{Supported Interaction Modalities}

The prototype supports three interaction modalities with holograms for (re)positioning them in 3D space. In the following, we will describe how in each modality a hologram can be moved around and rotated, and how, if applicable, users can switch between a rotation mode and a movement mode. In order to keep it simple we will refer to the modality, which utilizes mid-air gestures recognized by HoloLens sensors as hPinch, and the modalities, which utilize a smartphone's interaction capabilities as mTouch and mTiltTouch. hPinch: When this interaction modality is selected, the user can place the hologram by using the tap-and-hold gesture (natively) supported by the HoloLens device [13]. To perform a tap-and-hold gesture, the user has to gaze at the hologram, grab it (or pinch it) with his thumb and forefinger, and "drag" the hologram into place. The rotation of the hologram can be adjusted identically after performing an air-tap gesture [13] on the hologram, which we implemented as an easy way to switch between the movement and rotation mode. The hologram will then change its color to indicate the activated mode.

mTouch: In this interaction modality the user can place and rotate the holograms by using the touch screen layout on the smartphone as presented on the left in Figure 3. The three-dimensional position of the hologram in the user's perceived environment can be adjusted by touch and moving the finger tip within the upper section (in relation to the current viewing angle defined by the HoloLens's position/orientation in space) (Figure $3 \mathrm{~A}$ ) of the GUI. In the lower section of the layout, holograms can be rotated asynchronously and simultaneously by interacting with a joystick-like GUI element (Figure $3 \mathrm{~B}$ ).

mTiltTouch: This modality combines the touch controls from interaction mode mTouch with tilt control, utilizing the mobile device's accelerometer and Gyroscope sensors. While the hologram's position can be adjusted via touching on the touch screen of the smartphone (Figure $3 \mathrm{C}$ ), its rotation can be modified by physically tilting the smartphone along a desired axis.

\subsection{Software and Implementation}

For communication between the mobile app and the HoloLens application, the MQTT protocol was used. MQTT was declared to be a standard protocol for the internet of things (IoT) [17] and allows clients to publish messages under certain topics which are distributed via a broker, such as the Eclipse Mosquitto [8], which was used in this project to transmit low-latency messages between the two clients. Transmitted messages contain commands for position and rotation adjustments which were broadcast under separate topics. The HoloLens application was developed with the game engine Unity3d. The basic interaction methods such as gaze and gesture were implemented by using prefabs and scripts from MixedRealityToolkit ${ }^{2}$. The MQTT client was implemented using a modified M2MQTT library and assets that can be found on GitHub ${ }^{3}$.

The mobile app for the Android OS was developed in the Android Studio IDE. For each Interaction Method, an optimized touch screen layout was implemented (Figure 3). The app can be used to switch between the interaction modes described in Section 3.1 and translates sensor inputs from the touchscreen and tilt sensors into commands that are published over an integrated MQTT client.

\section{USER STUDY}

In order to explore users' experiences when interacting in a nearfuture mixed-material public space we setup a user study environment, utilizing the aforementioned prototype as a probe and invited users to try out all three interaction techniques enabled by the probe.

\footnotetext{
${ }^{2}$ Microsoft Mixed Reality Toolkit (MRTK) https://github.com/Microsoft/ MixedRealityToolkit-Unity

${ }^{3}$ M2MQTT for Unity https://github.com/gpvigano/M2MqttUnity
} 


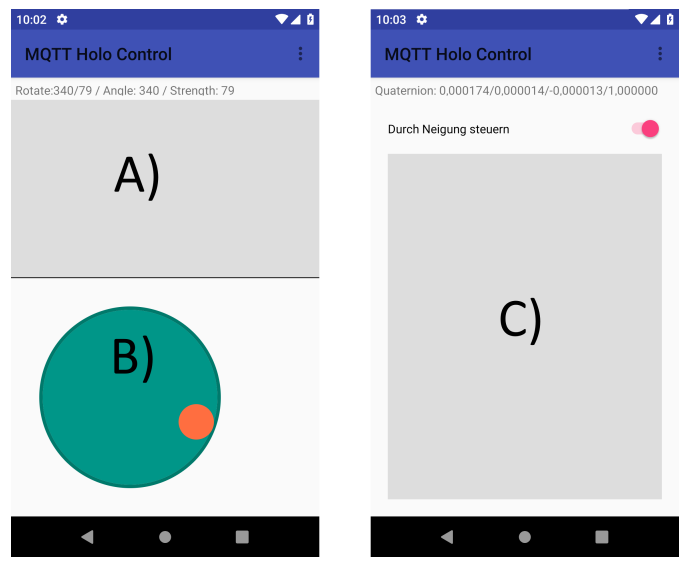

Figure 3: GUI layouts of the mobile app used for the interaction modalities mTouch (left) and mTiltTouch (right).

Four researchers conducted the study. One of the researchers was responsible to welcome and interact with the participants in case they needed help throughout the study and conduct the post-hoc interview. Two researchers observed each participant with one of the researchers observing participants directly while the other researcher observed the mixed-reality view, including the hologram, which was captured by the HoloLens and streamed on a separate monitor. The fourth researcher was responsible for the technical setup.
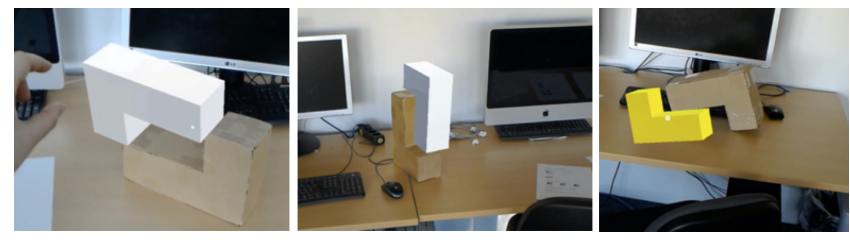

Figure 4: Screenshots taken from the HoloLens's live stream displayed at the supervisor PC.

\subsection{Participants and Apparatus}

Twelve participants $(6 \mathrm{f}, 6 \mathrm{~m})$ were recruited at a university campus. All participants were students with varying majors including Chemistry, Economics, and Computer Science. The study was conducted in a public terminal room in the computer science faculty. A first generation Microsoft HoloLens, a state-of-the-art Android Smartphone (i.e., Samsung's S7 edge model), and an L-shaped cardboard box were utilized in the study (see Figure 4).

\subsection{Procedure}

Each session started with welcoming the participants and providing them with an introduction to the topic of the study (i.e., future spaces inhabited with holograms and physical artifacts). This was done by showing them the official promotional video for the $\mathrm{Mi}$ crosoft HoloLens, which demonstrates some of Microsoft's visions of how the HoloLens could be used in the future. Then participants were asked to read an instructions document which described the task and illustrated the three interactions techniques (i.e., $m$ Touch, mTiltTouch, and hPinch), which were supported by the prototype they would be asked to try out. Following that, participants were allowed to try out each interaction technique until they felt comfortable. Afterwards, participants were asked to complete a task with each interaction technique in counterbalanced order.

The task of the participants was a "Put that hologram there" task. Participants were shown an image of how the physical L-shape cardboard box should be combined with the L-shaped hologram, which they could perceive through the HoloLens device. Participants were able to move and rotate the hologram in space. They were also allowed to directly manipulate the physical counterpart. The researcher who was observing the HoloLens's video-stream decided when the task was successfully completed. Participants were instructed to "think aloud" throughout the sessions. Participants took in average with each modality around 4 minutes to successfully complete their task. Overall, the study took for each participant about 45 minutes, including the semi-structured interview, which was conducted at the end with each participant, querying for example participants' anticipated expectations of a future where holograms were pervasively displayed.

\subsection{Results}

All of the data was collected through observations. The main analysis consisted of a thematic analysis [2], which included the creation of mind maps for each participant based on all observations, including participants' utterances during the "think aloud" protocol [7], which the researchers identified as important with respect to the research objectives (i.e., exploring potentials and limitations of hologram usage in future mixed-material public and urban spaces).

4.3.1 Results of quantifiable observations. In addition, the two researchers who were responsible to make notes throughout the study used predefined templates for their notes, which included to count the number of times participants changed their proximity to the desk, moved around the desk without changing their distance, how often and how they interacted with the physical counterpart, etc. Figure 5 presents frequency plots for these quantifiable observations separated by interaction technique. Our intention was to be able to identify possible differences in usage. Overall, participants didn't behave clearly different when using the interaction techniques. In all modalities participant interacted similarly often with the physical counterpart. The movement patterns of users moving towards and away from the desk, as well as, how they changed their orientation towards the desk were similar in all interaction modalities. Only when using the hPinch modality, they switched more often between the orientation change mode and the movement mode. The quantifiable results should be interpreted carefully, since the participants were not instructed to care for performance in completing the tasks and since our main research goal was not to compare traditional usability of the three interaction techniques.

4.3.2 Results of qualitative thematic analysis. Figure 6 presents the results of the main qualitative thematic analysis as a "model", which resulted from analyzing all mindmaps from all 12 participants and identifying the most common theme(s) discussed. The general model captures a dilemma which is experienced when participants 


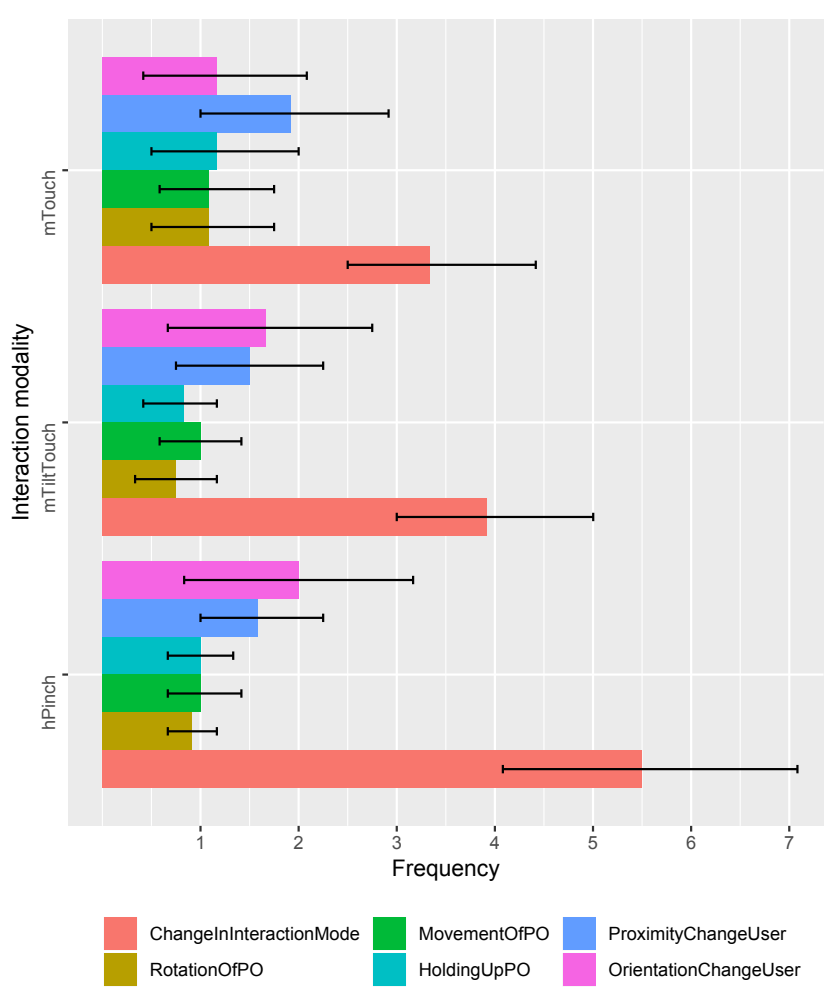

Figure 5: Overview of quantifiable observations describing interaction behavior during completing the task with the three different modalities. PO refers to physical object. Interaction mode refers to changing the "mode" from rotating to moving the hologram and vice versa. (Error bars denote 95\% confidence intervals)

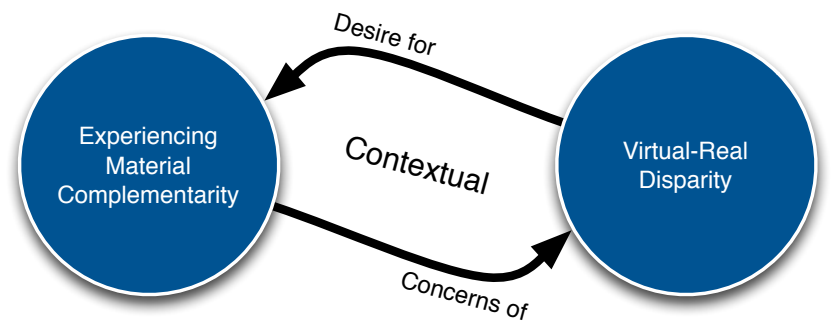

Figure 6: To a minimal size diverged model describing the two main interrelated themes emerged in the qualitative analysis.

interact in a public mixed-material space. The model describes how on the one hand side there is a desire for experiencing material complementarity, which is enabled in a mixed-material environment (i.e., an environment where participants can interact with holograms, physical objects and potential combinations). Participants stated, for example, "The combination of hologram and reality is really cool", "I like the combination of holograms and the real world", and "It's somehow cool to move something around that is not really there". On the other side, participants were concerned about distinguishing what is real and what is virtual. For example, one of the participants stated as a problem "Differentiating between reality and holograms will be more and more difficult with better holograms". The same participant also argued that "One might miss seeing a car driving around a corner because one is engaged in interacting with a hologram using a mobile". Another participant was also concerned that if head mounted displays and holograms would further improve, that the interactions with other people might decrease and that "There is a danger that people might push aside reality during real experiences, such as city tours".

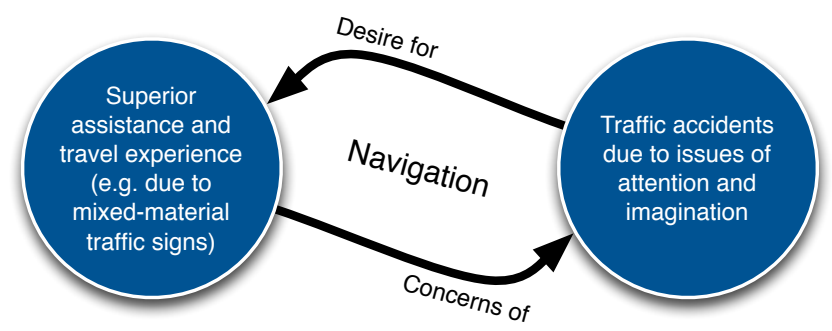

Figure 7: One example of applying the model in Figure 6, which resulted from the thematic analysis to navigation as an application context.

Participants often highlighted that the application and usage of holograms should be contextual. For example, some argued that in everyday life the usage of holograms and interactions with holograms might disrupt social interaction. Therefore, holograms should be used at home, at workplaces, and potential safe spaces. Participants also argued that when people use holograms in everyday life it should be possible to opt out. Participants also mentioned that holograms would be great to display ads but that it was important to use ads carefully and not excessively.

There were also ideas of single participants, which we found intriguing, such as one participant arguing that holograms would be great to use in smarthomes and that one could for example illustrate virtual light switches. Another participant argued that she could imagine having a "holographic" pet at home. Another one stated that holograms would be great to use in combination with menus in restaurants. Interestingly many of the participants (6) provided navigation as an example context to express their desire and concerns. Figure 7 illustrates the dilemma of interacting in a mixed-material public space for the navigation context. One participant expressed their concern for distraction by stating " $I$ would be afraid of being hit by a car". Another participant suggested that "a hologram in front of traffic lights could cause one to miss a red traffic light". But participants also suggested that holograms could be used to mark emergency exits, provide navigation assistance for bicyclists, and help personalize navigation instructions.

\section{DISCUSSION}

We have argued that in a popular near-future vision, enabled by mobile devices such as smartphones and head-mounted displays, people would have interactive encounters with holographic and real objects in public and urban spaces. We were interested in 
how these mixed material interactions and spaces would impact peoples' interaction experiences and anticipated future benefits and limitations of such a technological transformation.

Therefore, we created a setup for experience prototyping in which we combined a HoloLens and a smartphone, and implemented multimodal interaction techniques, which we considered paradigmatic in terms of what research in mobile HCI has studied in previous work (e.g. touch vs. tilt vs. gesture) and thus capturing the "zeitgeist" of interaction techniques enabled by today's mobiles.

The user study results have highlighted a dichotomy of expectations and experiences, which is well captured by the result of the qualitative thematic analysis. Participants enjoyed interacting in a mixed-material setup, which allowed them to move holograms in the air and through physical objects. We have observed that all but one participant not only interacted with the hologram but also moved, rotated, and held up the physical cardboard box to complete their tasks. On the other hand, participants voiced their concerns of a disparity of real and virtual, with participants bringing up most often traffic related examples to illustrate how dangerous it could become if people were asked to move in a mixed-material environment with holograms potentially distracting from real danger or encouraging a decoupling from reality and social interactions with others nearby. There was also concern that through technology progress and holograms becoming indistinguishable from real objects and the (dis)parity paradox would increase and potentially be more harmful or dangerous, since people would not be able to differentiate what is made of real materials and what only looks like being made of real material but is in truth intangible.

Interestingly $\mathrm{HCI}$ as a discipline has had similar controversial discussions with the notion of affordances (e.g., $[10,16])$ and how there can be a dilemma when things displaying perceivable but not real affordances (e.g., graphical 3D buttons on flat screens) invite "fake" actions because one can not really push for example a digital button on a flat screen even if one perceived that affordance.

It seems as if we need to be much more careful in how we disrupt affordances in a mixed material space than with graphical designs on screens by simulating material qualities which are non-existent and thus intangible, since the consequences in mixedmaterial spaces are potentially much more severe.

\section{CONCLUSION}

In this paper, we have reported on a user study to explore future interaction scenarios and to probe potential near-future mobile interaction experiences enabled by intangible holographic and tangible physical objects "co-existing" in public spaces. Our motivation is both technology-driven and (im)materiality-critical, aiming to predict the impact of a digital revolution on the face of urban space and consequent public interactions. Results of the described user study and especially results of the qualitative thematic analysis have put emphasis on what may seem obvious (i.e., there will be conflicts when holographic and physical things co-exist in urban space), including insights into a need to regulate and mark future holograms as intangibles, as one way to address the desire of participants to experience material complementarity of the virtual and real, and at the same time to address concerns of a disparity of the perceived materials and consequently between what seems real vs. what is real. While one way to control the virtual/material relationship is in looking into non-visual complementary interaction modalities, such as virbro-tactile feedback, for most humans vision and action is tightly connected (e.g., [1]) and we may have to also invest in empirical research to create new gestalt theories/principles, which are applicable to mixed-material environments.

\section{REFERENCES}

[1] Ilhan Aslan, Martin Murer, Verena Fuchsberger, Andrew Fugard, and Manfred Tscheligi. 2013. Drag and Drop the Apple: The Semantic Weight of Words and Images in Touch-based Interaction. In Proceedings of the 7th International Conference on Tangible, Embedded and Embodied Interaction (TEI '13). ACM, New York, NY, USA, 159-166. https://doi.org/10.1145/2460625.2460650

[2] Virginia Braun and Victoria Clarke. 2006. Using thematic analysis in psychology. Qualitative Research in Psychology 3, 2 (2006), 77-101.

[3] Bernard C. Kress and William J. Cummings. 2017. 11-1: Invited Paper : Towards the Ultimate Mixed Reality Experience: HoloLens Display Architecture Choices. SID Symposium Digest of Technical Papers 48 (05 2017), 127-131.

[4] Julie Carmigniani, Borko Furht, Marco Anisetti, Paolo Ceravolo, Ernesto Damiani, and Misa Ivkovic. 2011. Augmented Reality Technologies, Systems and Applications. Multimedia Tools Appl. 51, 1 (Jan. 2011), 341-377.

[5] Paul Dourish. 2004. Where the action is: the foundations of embodied interaction. MIT press.

[6] Paul Dourish. 2017. The stuff of bits: An essay on the materialities of information. MIT Press.

[7] K Anders Ericsson and Herbert A Simon. 1984. Protocol analysis: Verbal reports as data. the MIT Press.

[8] Eclipse Foundation. [n. d.]. Eclipse Mosquitto An open source MQTT broker. https://mosquitto.org/

[9] Verena Fuchsberger, Martin Murer, and Manfred Tscheligi. 2013. Materials, materiality, and media. In Proceedings of the SIGCHI Conference on Human Factors in Computing Systems. ACM, 2853-2862.

[10] William W. Gaver. 1991. Technology Affordances. In Proceedings of the SIGCHI Conference on Human Factors in Computing Systems (CHI'91). ACM, New York, NY, USA, 79-84. https://doi.org/10.1145/108844.108856

[11] Douglas E. Holmgren. 1992. Design and Construction of a 30-Degree See-Through Head-Mounted Display. Technical Report. Chapel Hill, NC, USA.

[12] Chi-Jung Lee and Hung-Kuo Chu. 2018. Dual-MR: Interaction with Mixed Reality Using Smartphones. In Proceedings of the 24th ACM Symposium on Virtual Reality Software and Technology (VRST '18). ACM, New York, NY, USA, Article 79, 2 pages.

[13] Microsoft. 2018. Mixed Reality Gestures. https://docs.microsoft.com/en-us/ windows/mixed-reality/gestures

[14] Paul Milgram, Haruo Takemura, Akira Utsumi, and Fumio Kishino. 1995. Augmented reality: a class of displays on the reality-virtuality continuum. , 2351 2351 - 11 pages. https://doi.org/10.1117/12.197321

[15] Alexandre Millette and Michael J. McGuffin. 2016. DualCAD: Integrating Augmented Reality with a Desktop GUI and Smartphone Interaction. In 2016 IEEE International Symposium on Mixed and Augmented Reality, ISMAR 2016 Adjunct, Merida, Yucatan, Mexico, September 19-23, 2016. 21-26. https://doi.org/10.1109/ ISMAR-Adjunct.2016.0030

[16] Donald A. Norman. 1999. Affordance, Conventions, and Design. interactions 6, 3 (May 1999), 38-43. https://doi.org/10.1145/301153.301168

[17] Oasis. [n. d.]. OASIS Message Queuing Telemetry Transport (MQTT) TC. https: //www.oasis-open.org/committees/tc_home.php?wg_abbrev=mqtt

[18] Video retrieved from NotPercy203 on 20.03.2017. [n. d.]. Marshall McLuahn The Medium is The Message [1977] (Media Savant). https://www.youtube.com/ watch?v=UoCrx0scCkM

[19] Yvonne Rogers. 2012. HCI theory: classical, modern, and contemporary. Synthesis lectures on human-centered informatics 5, 2 (2012), 1-129.

[20] Erik Stolterman and Lars-Erik Janlert. 2017. Things That Keep Us Busy: the elements of interaction.

[21] Ivan E. Sutherland. 1968. A Head-mounted Three Dimensional Display. In Proceedings of the December 9-11, 1968, Fall foint Computer Conference, Part I (AFIPS '68 (Fall, part I)). ACM, New York, NY, USA, 757-764.

[22] Kristoffer Waldow, Martin Misiak, Ursula Derichs, Olaf Clausen, and Arnulph Fuhrmann. 2018. An Evaluation of Smartphone-based Interaction in AR for Constrained Object Manipulation. In Proceedings of the 24th ACM Symposium on Virtual Reality Software and Technology (VRST '18). ACM, New York, NY, USA, Article 69, 2 pages. https://doi.org/10.1145/3281505.3281608

[23] Jeongmin Yu, Jinwoo Jeon, Jinwoo Park, Gabyong Park, Hyung-il Kim, and Woontack Woo. 2017. Geometry-Aware Interactive AR Authoring Using a Smartphone in a Wearable AR Environment. In Distributed, Ambient and Pervasive Interactions, Norbert Streitz and Panos Markopoulos (Eds.). Springer International Publishing, Cham, 416-424. 